\title{
Acute onset of lower limb claudication as a manifestation of a spontaneous common iliac artery dissection
}

\author{
Lukasz Hapka', Grzegorz Halena ${ }^{2}$ \\ 'Department of General Surgery, Specialist Hospital in Chojnice, Poland \\ ${ }^{2}$ Department of Cardiac and Vascular Surgery, Medical University in Gdansk, Poland
}

\begin{abstract}
Isolated abdominal aortic dissection constitutes a relatively small percentage of all aortic dissections. In this paper we present a case of a 59-year-old man who reported to the hospital because of the sudden appearance of intermittent claudication of his left lower limb. After establishing the diagnosis the patient was qualified for endovascular treatment due to dissection involving the final I cm of his abdominal aorta and extending to his left common iliac artery with significant true lumen compression. Stent was implanted into the left common iliac artery with almost complete occlusion of the false lumen and expansion of the true lumen of his iliac artery. During the 70-month follow-up we achieved a good result with evidence of stent patency and no signs of expansion of the false lumen. The paper discusses the epidemiology, aetiology, diagnosis and treatment of isolated abdominal dissections.
\end{abstract}

Key words: isolated abdominal aortic dissection, iliac artery dissection, endovascular treatment, common iliac artery

Acta Angiol 2017; 23, I: 9-12

\section{Introduction}

Isolated dissections of peripheral arteries or dissections of the abdominal aorta are rarely encountered in everyday practice as opposed to more common type $A$ and $B$ aortic dissections extending peripherally. We describe a patient treated 6 years ago for spontaneous, symptomatic dissection with entry in his abdominal aorta just above the bifurcation and extending to the left common iliac artery (CIA). We made the decision that the endovascular repair should only be limited to the affected left $\mathrm{CIA}$, without covering the entry in the atherosclerotic plaque just above aortic bifurcation. The patient has been followed for over 6 years without signs of dissection progression supporting our initial decision to avoid implantation of stent into the aorta and its possible downward migration.

\section{Case study}

A 59-year-old active smoker suffering from hypertension and coronary artery disease was admitted to the Department of Cardiac and Vascular Surgery with sudden onset of left lower limb claudication. Preoperative workup included computed tomography angiogram (CTA), which revealed dissection of the left CIA with entry tear in the abdominal aorta; the false lumen however almost completely occluded the true lumen of the CIA (Fig. I).

Based on CTA he was qualified for an endovascular intervention; it was decided to treat only iliac dissection without closing the primary tear in the abdominal aorta just above the ostium of the left CIA. After the introduction of a short $6 \mathrm{~F}$ sheath and confirming with angiography a significant iliac dissection, a self-expand-

Address for correspondence: Lukasz Hapka, Department of General Surgery, Specialist Hospital in Chojnice, Leśna I0,

89-600 Chojnice, Poland, tel.: +48 5239565 8I, e-mail: hapka@gumed.edu.pl 

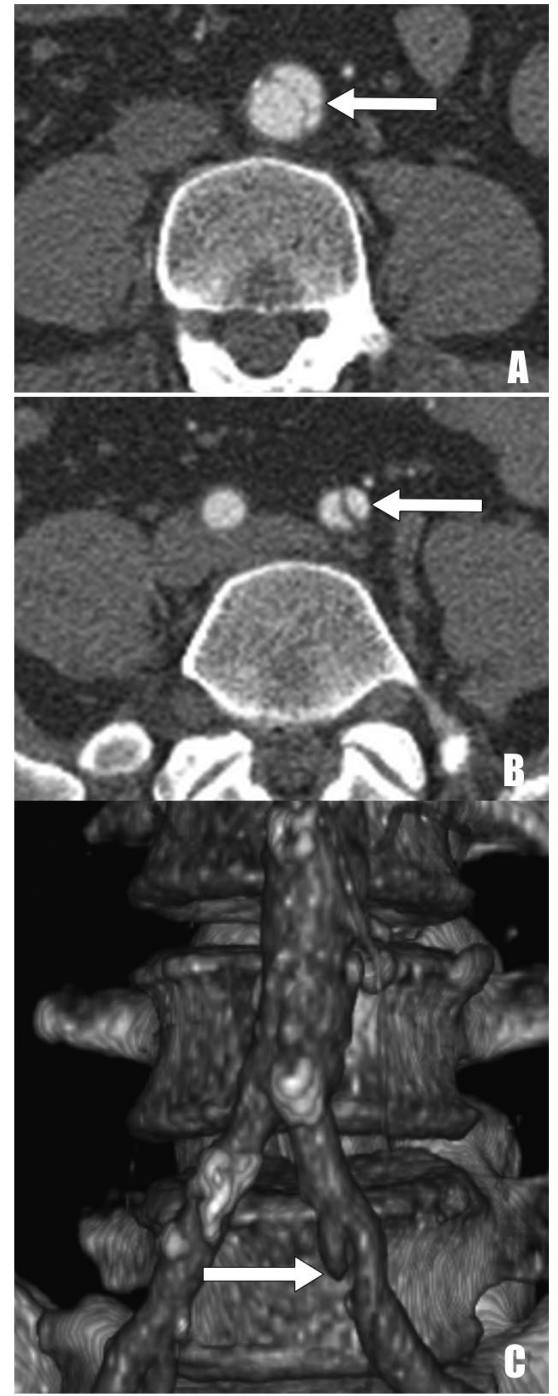

Figure I. A. Arrow points to the false lumen in the abdominal aorta; B. Arrow points to the false lumen in the CIA; C. Reconstruction of the false lumen in the CIA

able stent was implanted (Zilver $10 / 60 \mathrm{~mm}$, Cook Medical, Bloomington, IN, USA) and postdilated with an $8 \mathrm{~mm}$ angioplasty balloon. Completion angiography showed the exclusion of a false lumen (Fig. 2). Longterm follow-up demonstrated patency of the stent (Fig. 3), freedom from claudication and stable condition of the aortic entry tear not covered with an iliac stent.

\section{Discussion}

Isolated dissections of the abdominal aorta (IAAD) constitute about $1.1-4 \%$ of all aortic dissections $[I, 2]$. In the majority of aortic dissections the entry tear is located in the ascending $(62-70 \%$ of cases) or descending aorta (in the remaining $30-38 \%$ of cases) at the level of the left subclavian artery (type $A$ and $B$ Stanford classification respectively) [3, 4].

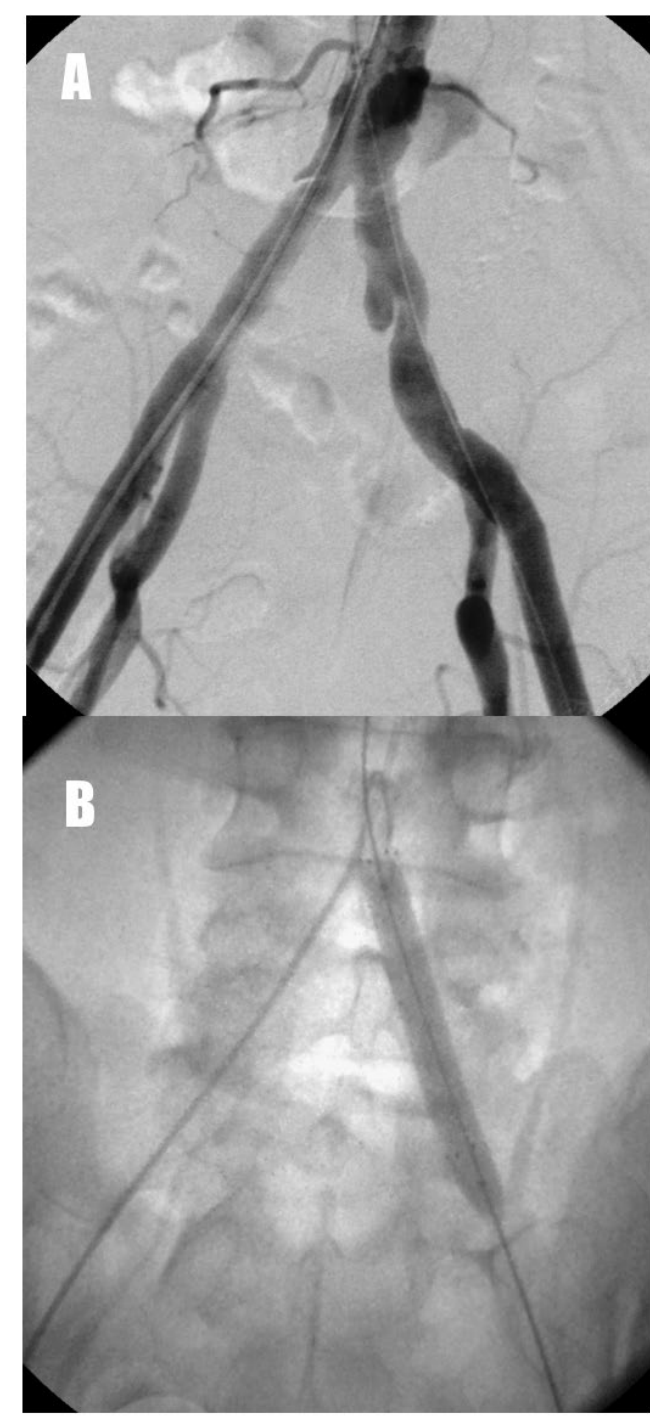

Figure 2A. Intraoperative angiography demonstrating entry and re-entry of dissection; B. Dilatation of an implanted $10 \mathrm{~mm}$ stent with an $8 \mathrm{~mm}$ balloon

Isolated dissections of the abdominal aorta are extremely rare, it is speculated that majority of them are iatrogenic and result from injury to the aortic wall during endovascular procedures [5]. Both penetrating injuries as well as high energy blunt trauma can lead to the disruption of the intima and subsequent dissection [6].

The role of hyperkinetic circulation in athletes and bikers has been raised in the literature [7]. Recurrent episodes of elevated blood pressure exceeding $200 \mathrm{~mm} \mathrm{Hg}$ during high-intensity workout can result in the degeneration of the intima leading to its disruption and dissection.

Any degenerative process involving tunica media can result in aortic dissection, predisposing individuals with genetic defects of collagen, elastin or smooth muscle cells to arterial dissection. Ehlers-Danlos syndrome (type IV), Marfan syndrome, Loeys-Dietz syndrome, 


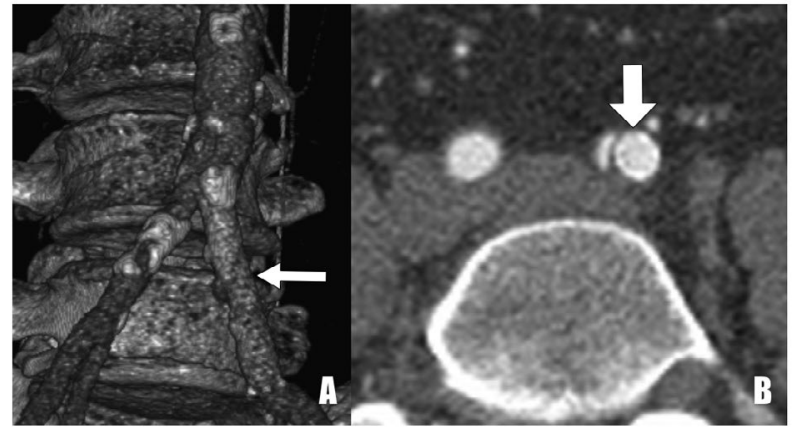

Figure 3A. Post procedural reconstruction of the distal aorta and CIA; B. Computed tomography angiogram — arrow points to the stent implanted to the $\mathrm{CIA}$, residual, narrow false lumen can be seen medially

fibro-muscular dysplasia [8, 9] are all examples of such genetic mutations.

Another example of genetic disorders leading to dissection is alfa-I-antitripsyn deficiency, which is an elastase inhibitor

Arterial hypertension is an example of an acquired disease, resulting in thickening of tunica media, decreasing number of smooth muscle cells and fibrosis of the intima. All those processes increase vessel wall stiffness and predispose to the possibility of vessel dissection.

Aortic dissection is manifested by acute pain $(82.4 \%)$, which is localized in the abdomen, back or chest $(68.7 \%, 62.5 \%, 41.2 \%$ respectively) [3].

Isolated claudication is present in $8.7 \%$ of cases. Abdominal pain, lower limb ischemia, are more frequent in IAAD than in type $B$ dissection [3].

CTA is often regarded to be the gold standard in the diagnosis of dissection; it is able to accurately identify true and false lumen, entry and re-entry, dilatation of the aorta and impending vessel rupture.

In cases of true abdominal location the entry is located between the renal arteries and the inferior mesenteric artery (in $50 \%$ of cases), while re-entry is usually in the CIA [I0].

In the presented case the entry was located $\mathrm{I} \mathrm{cm}$ above the aortic bifurcation, which according to Jonker et al. is the most rare entry site estimated to occur in $4.5 \%$ of IAAD.

Therapeutic decisions are based on the location, extent and potential instability of the dissection. Most of the authors recommend out-patient administration of the anti-platelet or anti-thrombotic drugs in the treatment of short dissections [ $1 \mathrm{I}-13]$ in asymptomatic cases.

Endovascular methods are often the first line treatment nowadays, and are especially effective for long lesions $[10,14]$. The aim of the therapy is implantation of a stent or stentgraft into the true lumen with the aim of collapsing the false lumen and redirecting the blood flow to the true lumen. The potential disadvantage of the method is occlusion of vital branches or bifurcation vessels.
We faced this clinical dilemma in our patient trying to find the balance between the simplest intervention (keeping the stent out of the aortic bifurcation) and a more radical attempt to close the entry located $\mathrm{I} \mathrm{cm}$ above aortic bifurcation which could imply typical EVAR procedure, implantation of a separate aortic stent or open aortic surgery.

Leaving uncovered entry just above the aortic bifurcation could result in persistent flow in the false lumen or even pressurized false lumen sac, however fearing that aortic stent just above the aortic bifurcation had a high potential of downward migration convinced us to choose spot stenting of the CIA only.

One might consider using a balloon-expandable stent in this location; however, due to the nature of the lesion (iliac dissection, not stenosis) we decided to use a self-expandable stent to better conform to the different diameters of the true lumen in the CIA. Additionally, due to the nature of the self-expandable stent we can expect further gain in the diameter of the true lumen with time, if the full expansion of the stent cannot be achieved during the initial procedure. Self-expandable stents exert constant outward radial force and balloon-expandable stents do not have this characteristic.

\section{Conclusions}

In the described case we decided to report not only the rare IAAD symptomatic dissection, but also excellent long-term outcome with stable aortic flap above stented CIA.

With increasing number of both symptomatic and asymptomatic aortic and peripheral arterial dissections diagnosed we face everyday clinical dilemma with either watchful waiting or endovascular intervention hoping to remodel the vessel and avoid possible future interventions. Treatment of late complications of aortic dissections (late aneurysmatic dilatation, narrowing of the true lumen) is not possible due to the thickening of the dissection membrane. Late remodelling is simply not 
effective with simple stent or stentgraft implantation, as the dissection membrane loses its elasticity after several weeks or months. Therefore the decision regarding conservative or interventional treatment has to be made upon patient presentation or soon thereafter.

We think that with limiting the extent of the procedure to CIA only, we achieved optimal and durable result without the need to invade aortic bifurcation. Leaving uncovered aortic entry tear can be questionable, and probably it would not be a good decision in case of a false lumen bigger than true aortic lumen.

During the follow up extending for more than 6 years we have observed complete exclusion of the false lumen, no symptoms of claudication and no new dissections, which supports our earlier decision of spot stenting the $\mathrm{CIA}$ only.

\section{References}

I. Graham D, Alexander JJ, Franceschi D, et al. The management of localized abdominal aortic dissections. J Vasc Surg. 1988; 8(5): 582-591, indexed in Pubmed: 3054172.

2. Hirst $A E$, Johns VJ, Kime SW. Dissecting aneurysm of the aorta: a review of 505 cases. Medicine (Baltimore). 1958; 37(3): 217-279, indexed in Pubmed: 13577293.

3. Trimarchi S, Tsai T, Eagle KA, et al. International Registry of Acute Aortic Dissection (IRAD) investigators. Acute abdominal aortic dissection: insight from the International Registry of Acute Aortic Dissection (IRAD). J Vasc Surg. 2007; 46(5): 913-919, doi: 10.1016/j.jvs.2007.07.030, indexed in Pubmed: 17980278.

4. Roberts CS, Roberts WC. Aortic dissection with the entrance tear in abdominal aorta. Am Heart J. 1991; $121(6 \mathrm{Pt} \mathrm{I})$ : 1834 1835, indexed in Pubmed: 2035406.
5. Weibull $H$, Bergqvist $D$, Jonsson $K$, et al. Complications after percutaneous transluminal angioplasty in the iliac, femoral, and popliteal arteries. J Vasc Surg. 1987; 5(5): 68I-686, indexed in Pubmed: 29528II.

6. Tuech JJ, Villapadierna F, Singland JD, et al. Blunt injury to the common iliac artery. Eur J Vasc Endovasc Surg. 2000; 20(I): 47-50, doi: $10.1053 /$ ejvs.2000.11 18, indexed in Pubmed: 10906297.

7. Cook PS, Erdoes LS, Selzer PM, et al. Dissection of the external iliac artery in highly trained athletes. J Vasc Surg. 1995; 22(2): 173-177, indexed in Pubmed: 7637118.

8. Akashi $\mathrm{H}$, Nata $\mathrm{S}$, Kanaya $\mathrm{K}$, et al. Spontaneous dissection of the iliac artery in a patient with fibromuscular dysplasia. Ann Vasc Surg. 2010; 24(7): 952.el3-952.el6, doi: 10.1016/j.avsg.2010.02.047, indexed in Pubmed: 20599351.

9. Ida $\mathrm{H}$, Taniguchi $\mathrm{N}$. Isolated common iliac artery dissection and aneurysm. BMJ Case Rep. 2016; 2016, doi: 10.1136/bcr-2016214989, indexed in Pubmed: 27030465.

10. Jonker FHW, Schlösser FJV, Moll FL, et al. Dissection of the abdominal aorta. Current evidence and implications for treatment strategies: a review and meta-analysis of 92 patients. J Endovasc Ther. 2009; 16(I): 7I-80, doi: 10.1583/08-260I.I, indexed in Pubmed: 19281293.

II. Sedivy P, El Samman K, Slais M, et al. A spontaneous isolated dissection of the external iliac artery in a roofer. Bratisl Lek Listy. 20I I; I I 2(7): 407-409, indexed in Pubmed: 21744738.

12. Teh LG, Sieunarine K, Van Schie G, et al. Spontaneous common iliac artery dissection after exercise. J Endovasc Ther. 2003; IO(I) 163-166, doi: 10.1177/152660280301000131, indexed in Pubmed: $\underline{12751950}$.

13. Thalhammer C, Aschwanden M, Blum B, et al. Unusual cause of intermittent claudication. Vasa. 2004; 33(4): 257-259, doi: $10.1024 / 030 \mid-1526.33 .4 .257$, indexed in Pubmed: 15623205.

14. Hirai S, Hamanaka Y, Mitsui N, et al. Spontaneous and isolated dissection of the external iliac artery: a case report. Ann Thorac Cardiovasc Surg. 2002; 8(3): 180-182, indexed in Pubmed: 12472404. 